\title{
Individual energetic processes efficiencies in a polycrystalline silicon PV cell versus electromagnetic field
}

OUEDRAOGO Adama ( $\sim$ adama1.ouedraogo@ujkz.bf )

Centre Universitaire Polytechnique de Kaya (CUPK)/Université Joseph KI-ZERBO https://orcid.org/0000-0003-2478-2521

\section{Mahamadi SAVADOGO}

Université Joseph Ki-Zerbo: Universite Joseph Ki-Zerbo

\section{Prince Abdoul Aziz HONADIA}

Université Joseph Ki-Zerbo: Universite Joseph Ki-Zerbo

\section{Sié KAM}

Université Joseph KI-ZERBO

\section{Dieudonné Joseph BATHIEBO}

université Joseph Ki-Zerbo

\section{Research Article}

Keywords: Polycrystalline silicon PV cell, Absorption efficiency, Thermalization efficiency, Thermodynamic efficiency, Fill factor, Conversion efficiency

Posted Date: August 16th, 2021

DOl: https://doi.org/10.21203/rs.3.rs-813811/v1

License: (9) This work is licensed under a Creative Commons Attribution 4.0 International License. Read Full License 


\title{
Individual energetic processes efficiencies in a polycrystalline silicon PV cell versus electromagnetic field
}

\author{
Adama OUEDRAOGO ${ }^{a, b *}$, Mahamadi SAVADOGO ${ }^{a}$, Prince Abdoul Aziz HONADIA ${ }^{a, b}$, Dieudonné Joseph BATHIEBO ${ }^{a}$, Sié \\ $\mathrm{KAM}^{\mathrm{a}}$ \\ ${ }^{a}$ Laboratory of Thermal and Renewable Energies, Department of Physics, Unit of Training and Research in Pure and applied Sciences, Université Joseph \\ KI-ZERBO, Ouagadougou, Burkina Faso; \\ ${ }^{b}$ Centre Universitaire Polytechnique de Kaya (CUP-Kaya), PO Box 232 Kaya, Burkina Faso \\ * Correspondence: adama1. ouedraogo@ujkz. bf
}

\begin{abstract}
The Photovoltaic (PV) system is often installed near the telecommunication antenna without takes account the performance degradation that the electromagnetic field can cause. The present work provides the recognition about the greatest losses occur which can cause the overall efficiency drop. In fact, the absorption and the thermodynamic processes are more sensitive to the variation of the electromagnetic field more than FF and thermalization processes in presence of the electromagnetic field. The absorption and thermodynamic mechanism are the main cause of the degradation of the polycrystalline silicon PV cell outputs. The PV cell having height base doping level to get a better resistivity to the electromagnetic field must be chosen to improve theses outputs. Then a low electromagnetic field zones must be searched to install the PV system improving its electrical production performance.
\end{abstract}

Keywords: Polycrystalline silicon PV cell, Absorption efficiency, Thermalization efficiency, Thermodynamic efficiency, Fill factor, Conversion efficiency.

\section{Introduction}

The solar energy mainly the photovoltaic (PV) energy is a real opportunity to mitigate the climate change problems. It can be used to compensate the weakness of the electric grid in the developing countries. The Sahelian strip presents a hight solar potential. However, the hight level heat is a serious difficulty for the PV systems[1]. But the PV cell in silicon mainly in polycrystalline silicon can be used in this strip because of its heat resistivity [1]. Those reasons cause an proliferation of the polycrystalline PV cell use often in the great capacity PV power plant as Zagtouli in Burkina Faso[2].

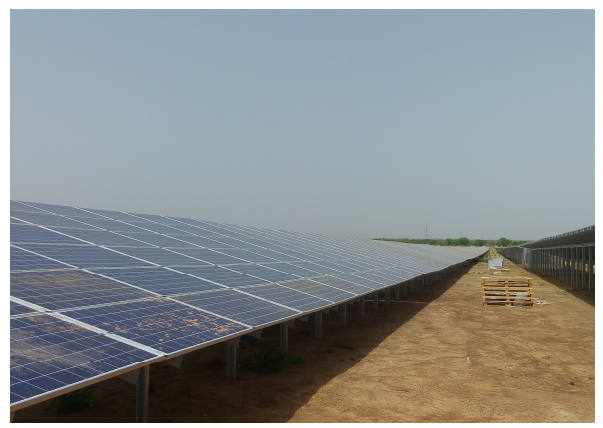

Figure 1: Zagtouli power plant $\left(33 M W_{c}\right)$ in Burkina Faso

The Zagtouli plant has a capacity of $33 M W_{c}$ and uses 129600 polycrystalline silicon PV modules of $260 W_{c}$. The energy produced presents almost $10 \%$ of the electricity needs of Ouagadougou, a city of 2.5 millions peoples environ [3]. Beside the development of the PV energy use, there is the proliferation of the technologies emitting the electromagnetic field in the environment as the base transceiver stations (BTS), the television (TV) and radios antennas, etc. Sometimes, the PV system is used in vicinity to provide the electricity for the operation of these technologies as shown in the figure 2

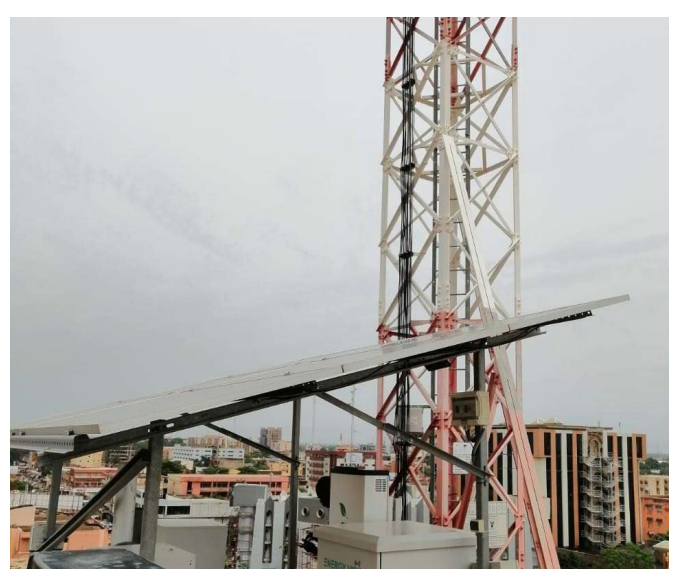

Figure 2: BTS electrified by a PV micro-grid

The proximity of the PV systems and the antennas can cause the interactions between the electromagnetic field produced and the PV systems where the silicon PV cell which is a PV system basic and fundamental component. The conversion efficiency of the silicon PV cell falls down when the electromagnetic field 
is great. However, the electromagnetic field is not attenuated during its crossing in the PV cell [4]. The current produced by PV cell increases versus the electromagnetic field increase while the electric voltage decreases [5]. Individually the magnetic field causes the reduction of the current and the increase of the voltage due to the deflection of the carriers charge [5]. The electric field causes and increases of the current [6, 7] due to the creation of the conduction current while the photovoltage decreases. The magnetic field of electromagnetic field from telecommunication antenna is weak but it can be not deleted as seen in some studies [8]. The electromagnetic field causes the division of the current in two components which are called leakage current and transferred current. The leakage current can cause the overheating of the $p n$ junction by Joule effect. Then the efficiency of the global process of the PV conversion decreases with the strong electromagnetic field strength. However, none of these studies set provides a deep explanation about the individual energetic mechanisms in the PV cell. It is then instructive to investigate the individual energetic processes in a polycrystalline solar cell versus electromagnetic field in order to recognize where the greatest losses occur and can cause the overall efficiency drop. The present work is about the efficiencies of the individual energetic processes in a polycrystalline PV cell versus electromagnetic field. In order to provides a deep comprehension about the PV cell efficiency fall down versus electromagnetic field from the calculated individual efficiencies, the devices were modelled using standard semiconductor device theory and the magneto-transport equation as well as the continuity equation are solved in the section of theories and methods. In the section of results and discussions, the influence of the electromagnetic field on the individual efficiencies of the PV cell will be provided. The last section of this study will be about the conclusion on the behaviour of the individual efficiencies and overall efficiency versus the electromagnetic field.

\section{Methods and Theories}

\subsection{Theories and assumptions}

The PV cell is the fundamental unitary component of a PV system. The low cost of the silicon and its non toxicity explained that it is the most abundant semi-conductor used in

the PV cell manufacturing. The device used in this study show an emitter ( $n^{+}$doping), a $p n$ junction, a base ( $p$ doping) and a rear face $\left(p^{+}\right.$doping). The modelling was restricted to $n^{+} / p / p^{+} \mathrm{PV}$ cell. The structure of a PV cell under electromagnetic field is given in the figure 3 .

The exponential doping gradient provides an constant strength electric field which pushes the minority carriers charge towards the $p-n$ junction. The high-low $p$ doping junction created in the interface a strong electric field named Back Surface Field $(B S F)$. It rejects the minority carriers back into the base of the PV cell and, thus, reduces the recombinations in the rear surface. The $B S F$ is incorporated by assuming a $p^{+}$region in the PV cell rear surface with $W_{h}=3 \mu \mathrm{m}$, and $N_{B}$ can reach until $5.10^{18} \mathrm{~cm}^{-3}$. $B S F$ can allow to overcome the recombination problem leading to the improvement efficiency [9].

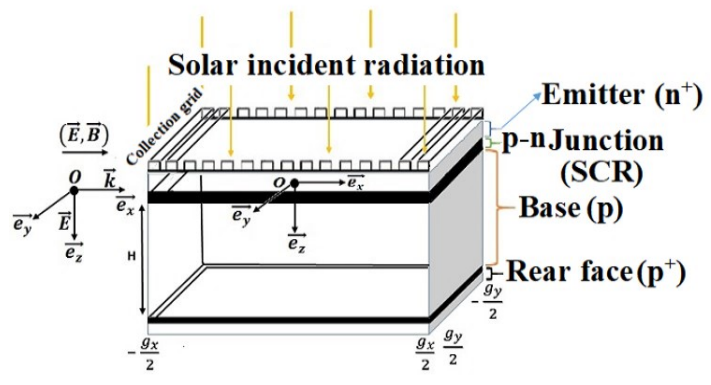

Figure 3: Illuminated PV cell under electromagnetic field

The PV system is installed under electromagnetic field. Then we will be able to extract an unitary PV cell to investigate the influence of the electromagnetic field on the individual efficiencies. The electromagnetic wave from radio wave $(9 \mathrm{kHz}$ to $3000 \mathrm{GHz}$ [ [10, 11] has an electric field and a magnetic field which are perpendicular in planar wave assumptions. It will be assumed a monochromatic planar wave with a straight line direction polarisation following the $(o x)$ axis $\left(\vec{k}=k \vec{e}_{x}\right)$ in the vacuum and according the direction of positives $z$.

The expressions of the electromagnetic field components are in the vacuum [12]:

$$
\vec{E}=E_{0} \cos (\omega t-k z) \vec{e}_{z}
$$

and

$$
\vec{B}=-B_{0} \cos (\omega t-k z) \vec{e}_{y}
$$

Where $B_{0}=\frac{E_{0}}{c}$ and $c$ is the light free space celerity. For the distance $(r)$ from electromagnetic field emission source to the PV system is great compared to the wavelength $(\lambda)$ i.e. $r \gg \lambda$, we have the far-field matching to Fraunhofer region of the antenna pattern, the power density decreases inversely as the square of the distance. It is the region where the antenna exchanges the energy with the external zone [13, 14]. The power density, $S$, in free space is given by the following equation [13, 14]:

$$
S=\frac{E_{0}^{2}}{Z_{0}}=H_{0}^{2} Z_{0}
$$

With $S$ : power density $\left(W \cdot m^{-2}\right)$ in a given direction $Z_{0}$ : the free space intrinsic impedance, $Z_{0}=120 \pi \Omega$. $E_{0}$ : electric field strength $\left(V . m^{-1}\right)(R M S) H_{0}$ : magnetic field strength $\left(A . m^{-1}\right)$ $(R M S) . \quad B_{0}=\mu_{0} H_{0}$ in Tesla $(T)$. The power density can be found in the far field as following at any given distance in any isotropic direction :

$$
S=\frac{P_{r} G_{i}}{4 \pi r^{2}}
$$

$P_{r}$ : power $(W)$ supplied to the radiation source, assuming a lossless system $G_{i}$ : gain factor of the radiation source in the relevant direction, relative to an isotropic radiator $r$ : distance $(m)$ from the radiation source. $P_{r} \cdot G_{i}$ is known as the equivalent isotropic radiated power (EIRP) which represents the power that a fictitious isotropic radiator would have to emit in order 
to produce the same field intensity at the receiving point [15]. The gain factor $G_{i}$ must be replaced by the product of $G_{r} . G_{a}$ [13, 14].

Table 1: Coefficients of the generation rate

\begin{tabular}{|l|l|l|}
\hline Reference antenna type & $G_{r}$ & Relevant applications \\
\hline Isotropic radiator & 1.0 & Radar, satellite, etc. \\
\hline Half-wave dipole & 1.64 & Television, $V H F$,etc. \\
\hline Short monopole & 3.0 & $L F, M F$, etc. \\
\hline
\end{tabular}

In the present case the gain of the antenna is expressed relative to a half-wave dipole: $G_{d}=G_{a}=2.15 d B$ [16, 17]. It will be assumed a fixed polarisation direction matching to PV system orientation tilt. The electromagnetic wave reaches the solar cell when $t=0$ and $x=y=0$. The electric field strength created in the $d$ distance from the transmitter is

$$
E_{0}=\frac{1}{2 r} \sqrt{\frac{P_{r} Z_{0}}{\pi}}
$$

The contribution of the base in photocurrent being greater than the emitter contribution [18]. This study is carried out assuming a ideal PV cell and its base as the center of the generation and recombination phenomena. It is also carried out in the theory of quasi-neutral base $(Q N B)[19,20]$. In this theory the intrinsic electric field in the base's region can be neglected. The radiation may be received or reflected by the semiconducting devices, or may be influenced the power conditioning electronics [21]. The effective length $L_{e f f}$ and the effective relative permittivity $\epsilon_{e f f}$ can be calculated using empirical formulate as following [21].

$$
L_{e f f} \cdot v_{r e s}=\frac{c}{2 \sqrt{\varepsilon_{e f f}}}
$$

Where $c$ is the light velocity in the free space and $v_{\text {res }}$ is the resonance frequency. The standard magneto-transport theory to describe the base electronic transport in PV cell provides [Betser 1995]

$$
\vec{J}_{n}=q D_{n} \overrightarrow{\operatorname{grad}} \delta(x, y, z)-\mu_{n} \vec{J}_{n} \times \vec{B}+q \mu_{n} \delta(x, y, z) \vec{E}
$$

The one dimensional $(1 D)$ assumptions according to the model provided by Betser et al.[22] is used to solve the mathematical equations. The base dimensions along the $x$ and $y$-axis are much larger than the base width $(H)$ following the $z$ axis. Then

$$
\frac{\partial \delta(x, y, z)}{\partial y}=\frac{\partial \delta(x, y, z)}{\partial x}=0
$$

Where $q$ is the electron charge, $\delta(z)$ gives the excess minoritary carriers charge and $D_{n}$ is the diffusion coefficient

$$
J_{n z}=q D_{n}^{*} \frac{\partial \delta(z)}{\partial x}+q \mu_{n}^{*} E_{0} \delta(z)
$$

With $D_{n}^{*}=\frac{D_{n}}{1+\left(\mu_{n} B_{0}\right)^{2}}$ the electron diffusion coefficient depending to the magnetic field and $\mu_{n}^{*}=\frac{\mu_{n}}{1+\left(\mu_{n} B_{0}\right)^{2}}$ the electron mobility coefficient depending to the magnetic field.

\subsection{Excess minoritary carriers charge}

The carriers charge conservation equation named continuity equation in $1 D$ approximation of a $p-n$ junction polycrystalline silicon PV cell in steady-state is described based on one-diode model as [23, 24, 25]:

$$
\frac{\partial^{2} \delta(z)}{\partial z^{2}}+\frac{\mu_{n} E_{0}}{D_{n}^{*}} \frac{\partial \delta(z)}{\partial z}-\frac{\delta(z)}{L_{n}^{* 2}}=-\frac{1}{D_{n}^{*}} \sum_{i=1}^{3} a_{i} e^{-b_{i} x}
$$

With $a_{i}$ and $b_{i}$ the coefficients deduced from modelling from the generation rate for the $A M 1.5$ spectrum on the surface of the earth [26]. $L_{n}^{*}=D_{n}^{*} . \tau_{n}$. It is the diffusion length depending to the magnetic field. The solution of this differential equation is given by $\delta_{0}(x)$ the contribution in the density of minority carriers charge in excess of the PV cell in obscurity and $\delta_{I}(x)$ the contribution of the PV cell under solar illumination [1, 27]. Then the definitive solution is such as

$$
\delta(x)=\delta_{0}(x)+\delta_{I}(x)
$$

The density of minority carriers charge in excess is then provided by

$$
\delta\left(z, S_{f}\right)=e^{\alpha z}\left[A\left(S_{f}\right) \cosh (\beta z)+B\left(S_{f}\right) \sinh (\beta z)\right]+\sum_{i=1}^{3} \gamma_{i} e^{-b_{i} z}(12)
$$

Where the different coefficients are given such as $\alpha=-\frac{\mu_{n} E_{0}}{2 D_{n}}$, $\beta=\frac{1}{2}\left[\left(\frac{\mu_{n} E_{0}}{D_{n}}\right)^{2}+\frac{4}{L_{n}^{* 2}}\right]^{\frac{1}{2}}$ and $\gamma_{i}=-\frac{a_{i}}{D_{n}^{*}\left[b_{i}^{2}-\frac{\mu_{n} E_{0}}{D_{n}^{*}} b_{i}-\frac{1}{L_{n}^{* 2}}\right]}$. The real constants $A$ and $B$ are provided by PV cell boundaries conditions equations [28] such as :

- In the $p n$ junction $z=0$

$$
\left.D_{n}^{*} \frac{\partial \delta(z)}{\partial z}\right|_{z=0}=S_{f} \delta(0)
$$

- In the rear face $z=H$

$$
\left.D_{n}^{*} \frac{\partial \delta(z)}{\partial z}\right|_{z=H}=-S_{b} \delta(H)
$$

The junction dynamic velocity is $S_{f}=S_{f_{j}}+S_{f_{0}}$. $S_{f_{0}}$ provides the intrinsic junction recombination velocity related to the losses of carriers charge at the junction interface and $S_{f_{j}}$ provides the flow of the minority carriers in excess crossing the $p-n$ junction. It is imposed by an external load and fixes the operating point of the PV cell[29]. For the ideal solar cell assumption, $S_{f_{0}}=0$.

\subsection{PV cell Electrical parameters}

The photocurrent is gotten by applied of the first Fick low such as

$$
J_{p h}\left(S_{f}\right)=q\left[\left.D_{n}^{*} \frac{\partial \delta\left(z, S_{f}\right)}{\partial z}\right|_{z=0}+\mu_{n}^{*} E_{0} \delta\left(0, S_{f}\right)\right]
$$


The rear side recombination velocity is obtained after the next equation solve [30]

$$
\left.\frac{\partial J_{p h}\left(S_{f}\right)}{\partial S_{f}}\right|_{S_{f} \rightarrow \infty}=0
$$

In the short circuit situation

$$
J_{s c}=\lim _{S_{f} \rightarrow \infty} J_{p h}\left(S_{f}\right)
$$

The total photocurrent density is not provided to the external load. One part called leakage photocurrent is leaked in the $p-n$ junction in the parasitic resistances. But the another part is transferred to the external load and can be named the transferred photocurrent. This second component is given as following using the equations 13 and 15 .

$$
J_{p h T}\left(S_{f}\right)=q S_{f} \delta\left(0, S_{f}\right)
$$

This photocurrent is taken for the next of the present study is the useful current provides by the PV cell to external load. The electrical voltage is obtained by applied of the Boltzmann low as :

$$
V_{p h}\left(S_{f}, s\right)=\frac{k_{b} T}{q} \ln \left[N_{B}(s) \frac{\delta\left(0, S_{f}\right)}{n_{i}^{2}}+1\right]
$$

Where $N_{B}(s)=10^{s} \mathrm{~cm}^{-3}$ is the doping level with $s$ from 14 to 18 and $k_{b}$, the Boltzmann constant. The accurate intrinsic carriers density $\left(\mathrm{cm}^{3}\right)$ depending to temperature [28, 31] is:

$$
n_{i}(T)=A \cdot T^{\frac{3}{2}} e^{-\left(\frac{E_{g}}{2 k_{b} T}\right)}
$$

Where $A=3.87 \cdot 10^{16} \mathrm{~cm}^{-3} \cdot K^{-\frac{3}{2}}$.

The electrical power is found by the following equation

$$
P_{p h}\left(S_{f}\right)=V_{p h}\left(S_{f}\right) \cdot J_{p h T}\left(S_{f}\right)
$$

The conversion efficiency $\left(\eta_{1}\right)$ is found from maximum power $\left(P_{p h_{\max }}\left(S_{f}\right)\right)$ obtained from previous equation and taking account the incident radiation under $A M 1.5$.

$$
\eta_{1}=\frac{P_{p h_{\max }}}{P_{\text {in }}}
$$

Where $P_{\text {in }}=1 \mathrm{~kW} \cdot \mathrm{m}^{-2}$

\subsection{Individual efficiencies}

The individual process are described by P. Wufer [32] according the figure 4

The absorption of the incident energy current is the first process [32]. Its efficiency is provided by

$$
\eta_{a b s}=\frac{J_{a b s}}{P_{\text {in }}}
$$

This absorption has to account for the photons with energy $E_{g}>$ $h \omega$, which are not absorbed by the PV cell. In the present paper, it will be assumed that each one photon absorption provides one

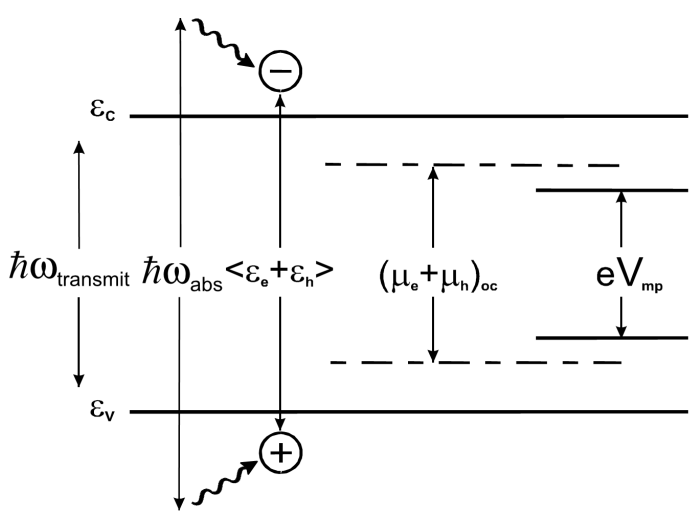

Figure 4: Individual processes representation

electron-hole pair only which contributes to the current at shortcircuit [32].

$$
J_{a b s}=\frac{J_{s c}}{e}\left\langle\hbar \omega_{a b s}\right\rangle
$$

For a PV cell under the $A M 1.5$ spectrum $\left\langle\hbar \omega_{a b s}\right\rangle=1.8 e V[32]$. The next process is about the thermalization of the electron-hole pairs. Its efficiency is expressed as [32]

$$
\eta_{\text {thermal }}=\frac{\left\langle\varepsilon_{e}+\varepsilon_{h}\right\rangle}{\left\langle\hbar \omega_{a b s}\right\rangle}
$$

With $\left\langle\varepsilon_{e}+\varepsilon_{h}\right\rangle=E_{g}+3 k_{b} T$ which is depending to the variation of the energy gap $E_{g}$. In presence of the electric field, $E_{0}$, the band gap edges are tilted. An electron motion inside a band gap a distance $z$ away from the edge and maintaining a constant total energy acquires a kinetic energy $q E_{0} z$ [33]. The states at the band edges are effectively broadened exponentially into the gap by an average deviation $\Delta E$ which depends on the electric field and can be shown by

$$
\Delta E_{g}=\frac{3\left(q \hbar E_{0}\right)^{\frac{2}{3}}}{2\left(m^{*}\right)^{\frac{1}{3}}}
$$

In presence of the magnetic field following the y direction $H_{y}$ in the PV cell, the electron motion in this direction is not affected, but the motion component in the transverse direction result in a periodic circular motion with pulsation (angular frequency):

$$
\omega_{c}=\frac{q H_{y}}{m^{*} c}
$$

$\omega_{c}$ is cyclotron frequency. The net shift of the energy gap is then [7, 33]

$$
\Delta E_{g}=\frac{1}{2} \frac{q \hbar H_{y}}{c}\left(\frac{1}{m_{c}^{*}}+\frac{1}{m_{v}^{*}}\right)
$$

$\hbar$ is the reduced Plank constant, $m_{c}^{*}$ and $m_{v}^{*}$ are respectively effective masses in the conduction and valence bands of the silicon semi-conductor. 
Among the processes, there is the thermodynamic process and its efficiency is given by

$$
\eta_{\text {thermo }}=\frac{e V_{o c}}{\left\langle\varepsilon_{e}+\varepsilon_{h}\right\rangle}
$$

In physics interpretation of this process, it can say that it provides the maximum chemical energy which can be extracted from the energy of the electron-hole pairs and it is giving the efficiency accounting for the difference between free energy and energy with a thermodynamic factor.

The last process is about the extraction of maximum electric power $\left(P_{\max }\right)$ compared to the ideal electric power $\left(V_{o c} . J p h_{s c}\right)$. It is named fill factor $(F F)$. Shunt $\left(R_{s h}\right)$ and $\left(R_{S}\right)$ series resistances in the PV cell can reduce the $F F$ below its ideal value. In the ideal condition, the PV cell has a great shunting resistance $\left(R_{s h} \rightarrow \infty\right)$ and the series resistance tends to null value $\left(R_{s} \rightarrow 0\right)[23,34]$. Then, the $F F$ will be written as [23, 34].

$$
F F=\frac{v_{o c}-\ln \left(v_{o c}+0.72\right)}{v_{o c}+1}
$$

With $v_{o c}=\frac{e V_{o c}}{k_{b} T}$.

In physics interpretation, the PV cell fill factor provides the ratio of the maximum power output of the cell to ideal electric power which is the product of the open-circuit voltage $V_{o c}$ and the short-circuit current $I_{s c}$ [23, 34].

The product of the previous four individual efficiencies set allows to find the overall efficiency expressed by

$$
\eta_{2}=\eta_{\text {abs }} \times \eta_{\text {thermal }} \times \eta_{\text {thermo }} \times F F
$$

In this study a comparison will do between $\eta_{1}$ and $\eta_{2}$ for the validation of the results obtained which will be discussed in the following section.

\section{Results and Discussions}

The electromagnetic field from antenna is composed by electric field and magnetic field. The different components are shown on the figures 5,6 and 7 .

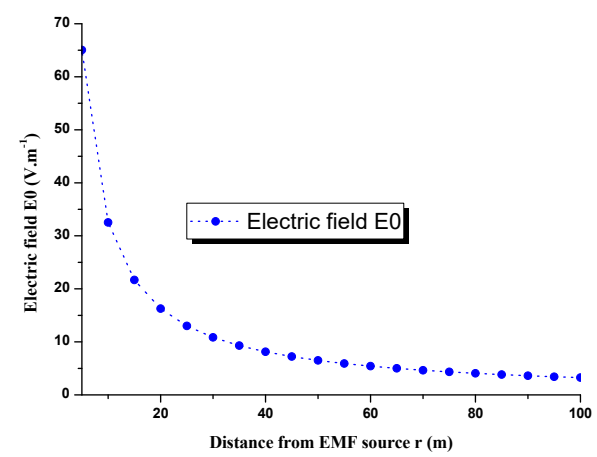

Figure 5: Electric field versus distance between emission source and PV cell

Electric field and magnetic field decrease versus the increase of the distance between the electromagnetic field source and

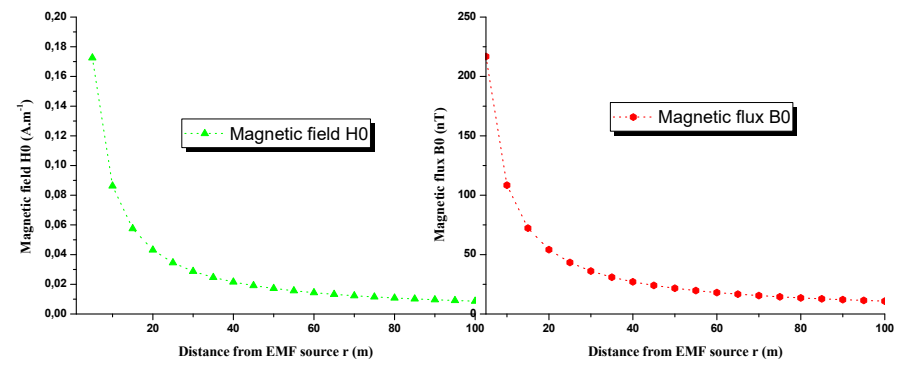

Figure 6: Magnetic field versus dis- Figure 7: Magnetic flux versus distance between emission source and tance between emission source and PV cell PV cell

the PV system. For the next of this work, the behaviour of the different parameters are studies in function of the distance. The electric and magnetic fields are great for the weak distance. These fields are weak for the great value of the distance. The photocurrent which the first parameter is presented in the figure 8 versus junction dynamic velocity for different distances.

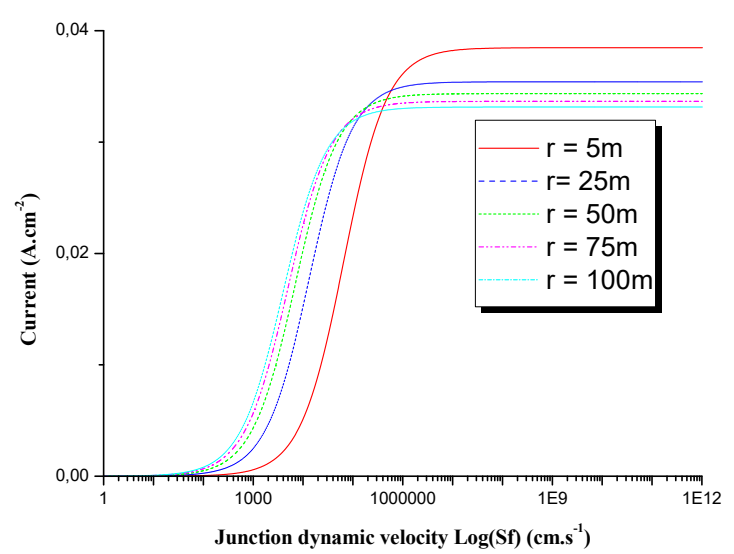

Figure 8: Current versus junction dynamic velocity for different distances

From the figure 8 , the current which is strongly depending to the junction dynamic velocity presents a great value in open circuit situation due to the electromagnetic field. It is also important in short circuit state for the weak distance compared to the great distance. That can be explained by the kinetic energy acquired by the carriers charge. In fact, the magnetic being weak (around 250nT), the electric field provides a sufficient energy to the carriers charge which in linear line can cross the junction and participate to the external current. However, in open circuit situation, the junction cross by the carriers charge causes the junction heating by Joule effect. From the linear side of the figure 8 , the rear face recombination velocity is deduced and shown in the figure 9 . 


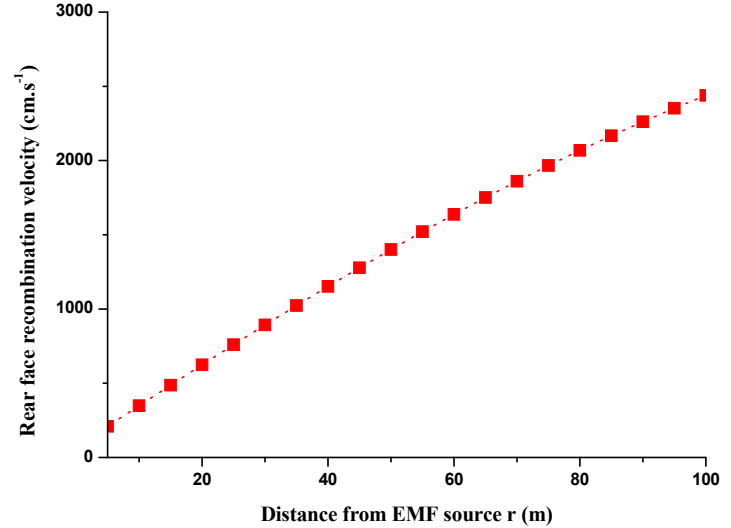

Figure 9: Rear face recombination velocity versus distance

A weakness of the rear face recombination velocity is observed when the distance is very low i.e. for the great electric field. The great magnetic field causes the deflection of the carriers charge. The direct consequence of the this deflection is the increase of the rear face recombination velocity and not its decrease when the distance is weak. It is then the electric field which is the cause of the decrease of the this velocity. That can explain the reduction of the rear face recombination. The electric voltage obtained thanks to Boltzmann low is presented in the figure 10

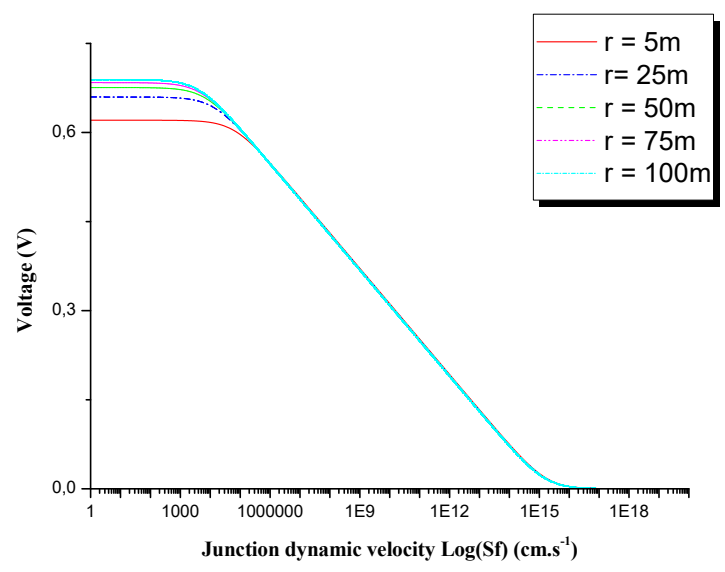

Figure 10: Voltage versus junction dynamic velocity for different distances

The voltage is important in open circuit state i.e. in low junction dynamic velocity value. However, this velocity decrease for the great electric field (low distance). This decrease is a confirmation about the important crossing of the junction by the carriers charge caused by the electric component of the electromagnetic field. However, the resistivity of the PV cell to electromagnetic field can be improved by the base doping level as shown in the figure 11 .

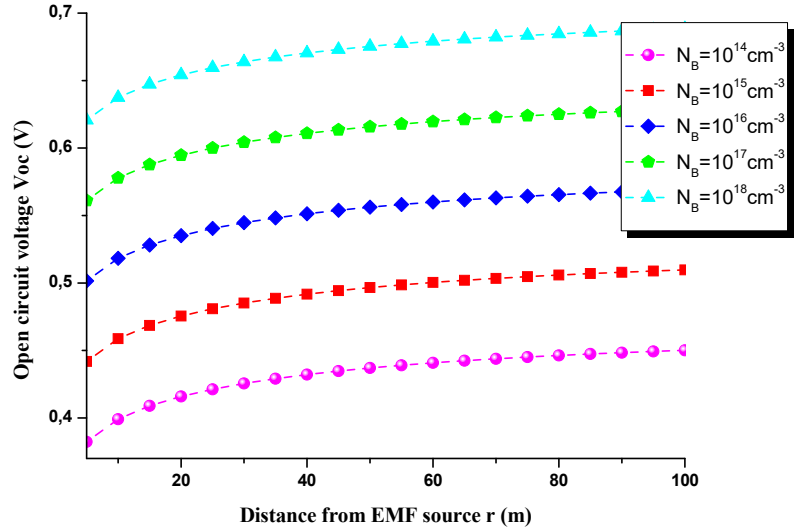

Figure 11: Open circuit voltage versus distance for different doping levels

For all doping level set, the open circuit voltage is weak in weak distance i.e. in great electric field. But it increase with the increase of the doping level from $10^{14} \mathrm{~cm}^{-3}$ to $10^{18} \mathrm{~cm}^{-3}$. The doping level can be then used to improve the voltage of the PV cell under electromagnetic field. The electric power is provided in the figure 12

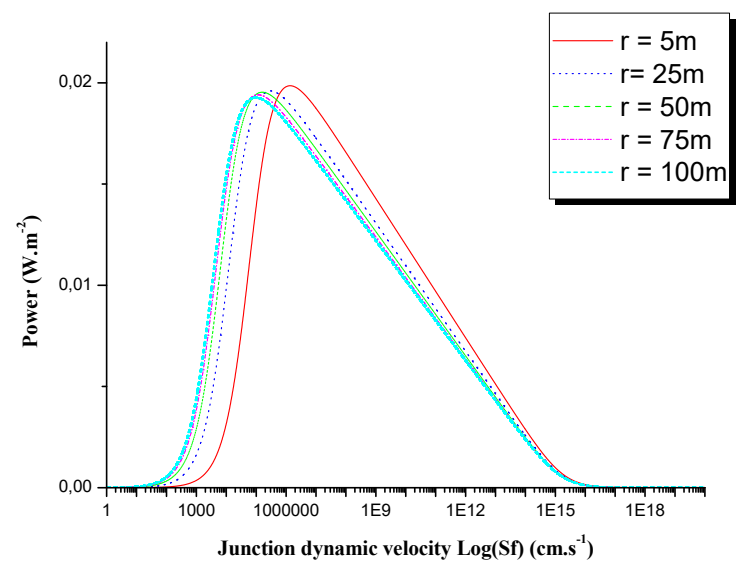

Figure 12: Electric power versus junction dynamic velocity for different distances

The electric power in figure 12 gives the influence of the electromagnetic field. The all distance values set, the electric power in the maximum power point is slightly shift from the normal operating point of the PV. That is explained by the crossing of the carriers charge in this state due to the influence of the electric field shifting the maximum power point toward the short circuit state. It is then a reason to install the PV system far to electromagnetic emission source, ideally in a distance more than $100 m$ according to the assumptions of this study. The maximum powers of the different distances are extracted and presented in the table 2 
Table 2: Maximum power and Overall efficiency

\begin{tabular}{|c|c|c|c|}
\hline$r(m)$ & $P_{p h_{\max }}\left(m W \cdot \mathrm{cm}^{-2}\right)$ & $\eta_{1}(\%)$ & $S_{f_{M P P}}\left(10^{m} \mathrm{~cm} \cdot \mathrm{s}^{-1}\right)$ \\
\hline 5 & 19.857 & 19.857 & 6.12 \\
\hline 25 & 19.602 & 19.602 & 5.46 \\
\hline 50 & 19.538 & 19.538 & 5.19 \\
\hline 75 & 19.411 & 19.411 & 5.04 \\
\hline 100 & 19.278 & 19.278 & 4.96 \\
\hline
\end{tabular}

Compared to the PV cell power without an electromagnetic field where the maximum power point is around $10^{4} \mathrm{~cm} \cdot \mathrm{s}^{-1}[1]$, in the present work, it is beyond $10^{4.5} \mathrm{~cm} \cdot \mathrm{s}^{-1}$. The PV conversion efficiency increase with the increase of the electromagnetic field. There is in this study a confirmation that an electromagnetic field mainly an electric field in low values assumptions can be used to improve the outputs of the polycrystalline silicon PV cell [25, 27]. The doping level affecting the outputs of the PV cell, some individual efficiencies are represented in the next paragraph.

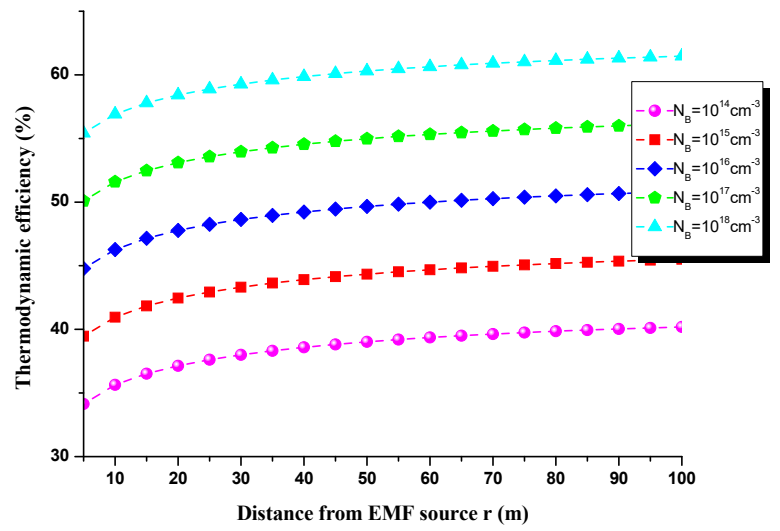

Figure 13: Thermodynamic efficiency versus distance for different base doping levels

The thermodynamic process is strongly depending to the open circuit voltage which also depends to the doping level. The better efficiency from conversion of the chemical energy to the electric energy is found for the greatest base doping level. However for the silicon matter, this doping level is low than $10^{19} \mathrm{~cm}^{-3}$ to avoid the degeneration of the semi-conductor [35]. When the PV cell is far from electromagnetic field emission source, there is an improving of the thermodynamic process.

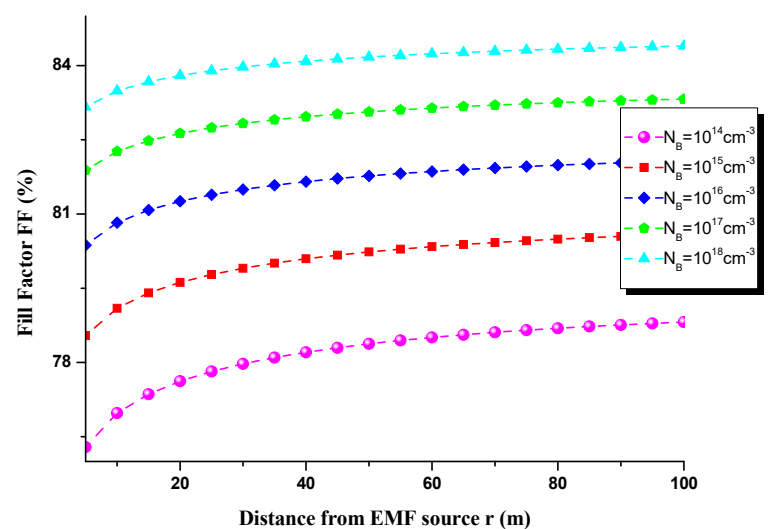

Figure 14: Fill factor versus distance for different base doping levels

From the figure 14, the $F F$ presents an improving far from electromagnetic field emission source. It is better also for the hight base doping level due to the improving of the open circuit voltage versus base doping level. The hight base doping level can be used to protect the PV cell against the electromagnetic wave from any source as well as against the temperature [1]. All the individual efficiencies set versus electromagnetic field are shown in the figure 15 .

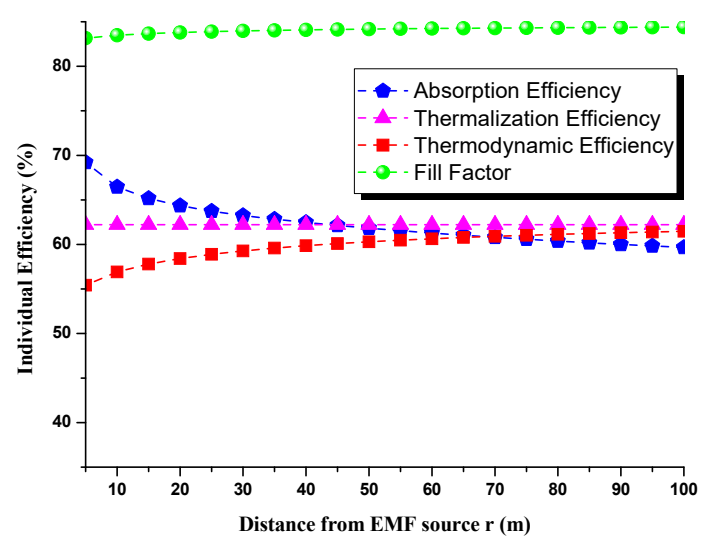

Figure 15: Individual efficiency versus distances

The thermalization process is not influenced by the electromagnetic field. The absorption process is improved with the great electromagnetic field. However, the $F F$ and thermodynamic are strongly affected by the increase of the electromagnetic field due to the dependence to the open circuit voltage. The absorption and the thermodynamic processes are more sensitive to the variation of the electromagnetic field more than FF and thermalization processes. The electromagnetic sensitivity of the absorption mechanism is due to the important $p n$ junction crossing by the carriers charge increasing the short circuit current. But, electromagnetic sensitivity of the thermodynamic is explained by its strongly dependence to the open circuit voltage 
which decreases with the increase of the electromagnetic field. The overall efficiency from the multiplication of these individual efficiencies is shown in the table 3 .

Table 3: Individual efficiencies versus distance from electromagnetic field emission source

\begin{tabular}{|c|c|c|c|c|c|}
\hline$r(m)$ & $\eta_{\text {abs }}(\%)$ & $\eta_{\text {thermal }}(\%)$ & $\eta_{\text {thermo }}(\%)$ & $F F(\%)$ & $\eta_{2}(\%)$ \\
\hline 5 & 69.205 & 62.222 & 55.417 & 83.163 & 19.845 \\
\hline 25 & 63.738 & 62.222 & 58.892 & 83.896 & 19.594 \\
\hline 50 & 61.829 & 62.222 & 60.300 & 84.173 & 19.526 \\
\hline 75 & 60.584 & 62.222 & 61.025 & 84.312 & 19.395 \\
\hline 100 & 59.664 & 62.222 & 61.468 & 84.395 & 19.258 \\
\hline
\end{tabular}

The absorption process presents an efficiency gap of $9.541 \%$ from $5 m$ to $100 m$ and the thermodynamic mechanism shows an efficiency gap of $6.051 \%$ while the $F F$ has a gap of $1.232 \%$. The overall efficiency decreases versus the increase of the electromagnetic field. The absorption and the thermodynamic processes are the mainly cause of the overall efficiency drop versus electromagnetic field. The overall efficiencies set are extracted and shown in the figure 16

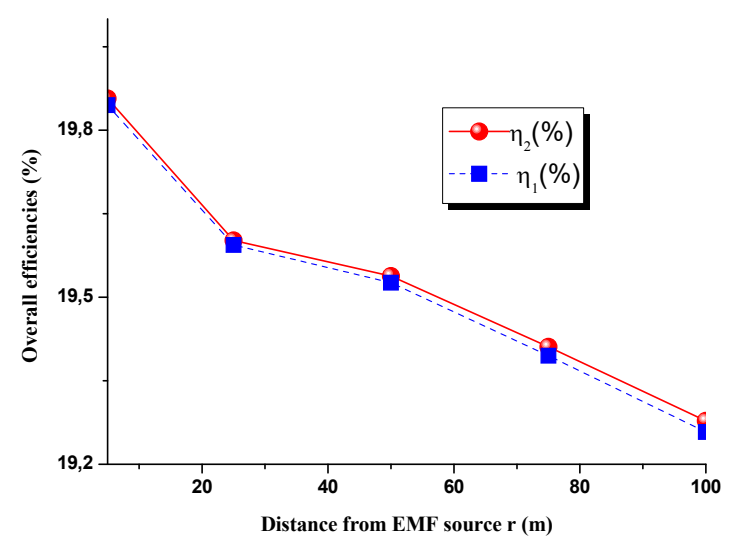

Figure 16: Overall efficiency versus distance

The both overall efficiencies have the same decrease slop degree when the PV cell is away from the electromagnetic field emission source. The increase observed when the electromagnetic field is great is not rentable to the PV conversion because of the Joule effect being very important in this situation. That can cause the heating of the $p n$ junction and degrading its performance. It can be advised to install PV system in distance more than $100 \mathrm{~m}$ and to chose a PV cell having height base doping level to get better resistivity to the electromagnetic field. In the table 4 the gap between $\eta_{1}$ and $\eta_{2}$ is presented.

The gap between the two overall efficiencies is very weak and the average gap is $\Delta \eta=\eta_{1}-\eta_{2}=\operatorname{Gap}(\%)=0.01311865$. That can prove the accuracy of the use of the individual efficiencies to recognize where the greatest losses occur and can cause the overall efficiency drop.
Table 4: Overall efficiencies $\eta_{1}$ and $\eta_{2}$

\begin{tabular}{|c|c|c|c|}
\hline$r(m)$ & $\eta_{1}(\%)$ & $\eta_{2}(\%)$ & $\operatorname{Gap}(\%)$ \\
\hline 5 & 19.857 & 19.845 & 0.01184028 \\
\hline 25 & 19.602 & 19.594 & 0.00723963 \\
\hline 50 & 19.538 & 19.526 & 0.01141451 \\
\hline 75 & 19.411 & 19.395 & 0.01558440 \\
\hline 100 & 19.278 & 19.258 & 0.01951442 \\
\hline
\end{tabular}

\section{Conclusion}

The electromagnetic field influence on the individual efficiencies is presented in this work. The electric component of the electromagnetic field is the real cause of the increase of the electrical current and the decrease of the electrical voltage. The electric component cause clearly the open circuit voltage drop. That can be explained by the important increase of the $p n$ junction crossing by the generated minoritary carries charge in the base. The current is so increase helped by the conduction current provides by the electric field. The base doping level can be used to improve silicon PV cell resistivity to the electromagnetic field. Among the individual energetic processes, the absorption and the thermodynamic processes are more sensitive to the variation of the electromagnetic field more than FF and thermalization processes. The electromagnetic sensitivity of the absorption mechanism is caused by the important pn junction crossing by the carriers charge increasing the short circuit current. But, electromagnetic sensitivity of the thermodynamic is due to its strongly dependence to the open circuit voltage which decreases according the increase of the electromagnetic field. Following these reasons, the installation of PV system in distance less than $100 \mathrm{~m}$ and is not advised. Then a PV cell having height base doping level to get better resistivity to the electromagnetic field must be chosen.

\section{References}

[1] Adama Ouédraogo, Bernard Zouma, Emmanuel Ouédraogoa, Lamoussa Guissoua, and Dieudonné Joseph Bathiébo. Individual efficiencies of a polycrystalline silicon PV cell versus temperature. Results in Optics, 2(4), August 2021.

[2] Babalwa Bungane. Burkina Faso unveils zagtouli solar power plant. ESI Africa https://www. esi-africa.com/industry-sectors/renewable-energy/ burkina-faso-unveil-zagtouli-solar-power-plant//2017.

[3] INSD Burkina Faso. $5^{e}$ recensement général de la population et de l'habitation (RGPH). Technical report, INSD Burkina Faso, 2020.

[4] A. Ouedraogo, V. D. B. Barandja, I. Zerbo, M. Zoungran, E. W. Ramde, and D. J. Bathiebo. A theoretical study of radio wave attenuation through a polycrystalline silicon solar cell. Turkish Journal of Physics, 2017.

[5] Serafettin Erel. Comparing the behaviours of some typical solar cells under external effects. Teknoloji, 11(3):233 - 237, 2008.

[6] and Mustafa A. Erel, Serafettin, K. E. Gazi, and C. Veli. The behaviour of a typical single-crystal si solar cell under high intensity of electric field. Solar Energy Materials $\mathcal{F}$ Solar Cells, 90:582 - 587, 2006.

[7] Serafettin Erel. The effect of electric and magnetic fields on theoperation of a photovoltaic cell. Solar Energy Materials $\mathcal{E}$ Solar Cells, 71:273 280, May 2002.

[8] A. Moissi, M. Zoungrana, A. Diallo, S. Mbodji, H. L. Diallo, A. Hamidou, M. Ndiaye, and G. Sissoko. Base transceiver station (BTS) antenna electric field influence on the space charge region in a silicon solar 
cell. Research Journal of Applied Sciences, Engineering and Technology, 7(2):2554-2558, 2014

[9] K. J. Weber, A. Cuevas, and A. W. Blakers. The influence of drift fields in thin silicon solar cells. Solar Energy Materials and Solar Cells, (45):151$160,1997$.

[10] ICNIRP. ICNIRP guidelines for limiting exposure to time-varying electric, magnetic and electromagnetic fields (up to $300 \mathrm{GHz}$ ). Health Physics, 74(4):494 - 522, 1998.

[11] Maxime Nicolas. Ondes et électromagnétisme. Dunod, 2009.

[12] J. P. Perez, R. Carles, and R. Fleckinger. Electromagnétisme - Fondements et applications. Dunod Paris, 4e edition, 2001.

[13] IUT. Evaluating fields from terrestrial broadcasting transmitting systems operating in any frequency band for assessing exposure to nonionizing radiation (question ITU-R 50/6). Technical report, IUT, ITU-R BS.2037, 2004.

[14] IUT. Evaluating fields from terrestrial broadcasting transmitting systems operating in any frequency band for assessing exposure to non-ionizing radiation. Technical report, IUT, ITU-R BS.1698, 2005.

[15] Agence nationale de radiofréquence (ANFR) France. Guide technique, modélisation des sites radioélectriques et des périmètres de sécurité pour le public, ANFR/DR 17- 53. ANFR, France, May 2018. ANFR/DR 175.

[16] Roland Guillaume (F5ZV depuis 1969). Le gain des antennes. Le Manuel Internet des Radioamateurs https: // f5zv. pagesperso-orange. $\mathrm{fr} / \mathrm{RADIO} / \mathrm{RM} / \mathrm{RM08/RM08a/RM08a05}$. html .2020 . F5ZV depuis 1969.

[17] fBinfographie. Les dipôles ou antennes doublets demi-onde. https: // www. leradioscope. fr/les-antennes/dipoles-demi-ondes 2016.

[18] J. Furlan and S. Amon. Approximation of the carrier generation rate in illuminated silicon. Solid-State Electronics, 28(12):1241 - 1243, 1985.

[19] J. G. Fossum, E. L. Burgess, and F. A. Lindholm. Silicon solar cell designs based on physical behavior in concentrated sunlight. Solid-State Electronics, 21:729 - 737, 1978.

[20] J. G. Fossum and D. S. Lee. A physical model for the dependence of carrier lifetime on doping density in nondegenerate silicon. Solid-State Electronics, 25(8):741-747, 1982

[21] E. Kancsar V. Schlosser G. Klinger M. Drapalik, J. Schmid. A study of the antenna effect of photovoltaic modules. European Association for the Development of Renewable Energies, Environment and Power Quality (EA4EPQ) , International Conference on Renewable Energies and Power Quality (ICREPQ'10) Granada (Spain), 23rd to 25th March, 2010, 1(8):371-375, 2010.

[22] Y. Betser, D. Ritter, G. Bahir, S. Cohen, and J. Sperling. Measurement of the minority carrier mobility in the base of heterojunction bipolartransistors using a magnetotransport method. Applied Physics Letters, 67(13):1883-1884, 1995

[23] M. A. Green. Accuracy of analytical expressions for solar cell fill factors. Solar cells, 7:337 - 340, 1982. Short Communication.

[24] A. L. Fahrenbruch and R. H. Bube. Fondamental of solar cell, photovoltaic energy conversion. Academic Press, 1983.

[25] Adama Ouedraogo, Thierry Sikoudouin Maurice Ky, Abdoulaye Compaore, and Dieudonné Joseph Bathiebo. Improvement in the silicon solar cell performance by integration of the electric field source in the solar cell under sunlight illumination. Arabian Journal for Science and Engineering, 44(7):6651 - 6657, May 2019.

[26] S. Noor Mohammad. An alternative method for the performance analysis of silicon solar cells. Journal of Applied Physics, 61(2):767 - 772, 1987.

[27] Adama Ouedraogo, Serge Dimitri Y. B. Bazyomo, Salifou Ouedraogo, Abdoul Razakou, and Dieudonné Joseph Bathiébo. Improvement of the silicon solar cell performance by integration of an electric field source in the solar cell or solar module system. Smart Grid and Renewable Energy, 9(12):285 - 298, December 2018.

[28] Adrian Kitai. Principles of Solar Cells, LEDs and Related Devices: The Role of the pn Junction, volume 2. John Wiley Sons Ltd, john wiley sons ltd edition, 2019.

[29] I. Zerbo, M Zoungrana, A. Ouédraogo, and D. J. Bathiebo. Effect of junction quality on the performance of a silicon solar cell. Journal of Fundamental and Applied Sciences, 9(2):1012 - 1026, 2017.

[30] H. L. Diallo, A. S. Maiga, A. Wereme, and G. Sissoko. New approach of both junction and back surface recombination velo cities in a $3 \mathrm{D}$ mod- elling study of a polycrystalline silicon solar cell. Phys. J. Appl. Phys, 42:203-211, 2008

[31] A. B. Sproul and M. A. Green. Improved value for the silicon intrinsic carrier concentration from 275 to 375 K. J. Appl. Phys., 70(2):846 - 854, 1991.

[32] P. Würfel. Physics of solar cells. Die Deutsche Bibliothek, Berlin, WileyVCH Verlag GmbH and Co. KGaA edition, 2005.

[33] J. Pankove Jacques. Optical processes in semiconductors. Dover publication Inc, New York, dover edition edition, 1971.

[34] M. A. Green. Solar cell fill factors: general graph and empirical expression. Solid-State Electronics, 24(8):788 - 789, 1981.

[35] H. Mathieu and H. Fanet. Physique des semiconducteurs et des composants électroniques. Dunod, 6 edition, 2009. 